\title{
Solar Neighbourhood Age-Metallicity Relation Based on Hipparcos Data
}

\author{
Akihiko Ibukiyama ${ }^{1,2}$ \\ ${ }^{1}$ Institute of Astronomy, University of Tokyo, 2-21-1 Osawa, Mitaka, Tokyo 181-0015, Japan \\ ${ }^{2}$ E-mail: aibukiya@optik.mtk.nao.ac.jp
}

Received 2003 October 1, accepted 2004 March 1

\begin{abstract}
We derive age-metallicity relations (AMRs) and orbits for the 1658 solar neighbourhood stars for which accurate distances are measured by the Hipparcos satellite. The sample comprises 1382 thin disk stars, 229 thick disk stars, and 47 halo stars according to their orbital parameters. We find a considerable scatter for thin disk AMRs along the one-zone Galactic chemical evolution (GCE) model. Orbits and metallicities of thin disk stars show no clear relation to each other. The scatter along the AMR exists even if stars with the same orbits are selected. We examine simple extensions of one-zone GCE models which account for inhomogeneity in the effective yield and inhomogeneous star formation rate in the Galaxy. Both extensions of the one-zone GCE model cannot account for the scatter in the age- $[\mathrm{Fe} / \mathrm{H}]-[\mathrm{Ca} / \mathrm{Fe}]$ relation simultaneously. We conclude, therefore, that the scatter along the thin disk AMR is an essential feature in the formation and evolution of the Galaxy. The AMR for thick disk stars shows that star formation terminated $8 \mathrm{Gyr}$ ago in the thick disk. As previously reported, thick disk stars are more Ca-rich than thin disk stars with the same $[\mathrm{Fe} / \mathrm{H}]$. We find that thick disk stars show a vertical abundance gradient. These three facts - AMR, vertical gradient, and $[\mathrm{Ca} / \mathrm{Fe}]-[\mathrm{Fe} / \mathrm{H}]$ relation - support monolithic collapse and/or accretion of satellite dwarf galaxies as likely thick disk formation scenarios.
\end{abstract}

Keyword: Galaxy: kinematics and dynamics

\section{Introduction}

The individual ages for solar neighbourhood stars are indispensable in research into the star formation history of the Galaxy. Twarog (1980) first derived the agemetallicity relation (AMR) for the disk in the neighbourhood of the Sun from $u b v y-\mathrm{H} \beta$ photometry. Edvardsson et al. (1993, hereafter E93) derived elemental abundances of 13 elements for 189 nearby long-lived disk dwarfs by using high resolution and high signal to noise spectroscopic data. Individual ages were derived photometrically from fits in the $\log T_{\text {eff }}-\log g$ plane. Due to metallicity measurements of high precision, E93 greatly improved the AMR, but ironically the resulting AMR clearly indicated a considerable scatter $(\sim 0.15 \mathrm{dex})$ in the $[\mathrm{Fe} / \mathrm{H}]$ of disk stars formed at any given time, implying that there is only a very weak correlation between age and metallicity. The scatter seems to be substantially larger than that which can be explained by observational errors. If the scatter is real, it would cause a serious difficulty for Galactic chemical evolution (GCE) models, because it is easy to fit the average run of the data, but difficult to explain such a large scatter (Pagel \& Tautvaišiene 1995, hereafter PT). In this article, we present newly derived AMR for 1658 stars and discuss the implications of the AMR.

\section{The Data}

\subsection{Observational Data}

Visual magnitudes, $B-V$ colours, and parallaxes were all taken from the Hipparcos catalogue (ESA 1997).
A certain number of stars in the Hipparcos catalogue suffer from uncertainties in parallax, binarity, and variability. Such stars are carefully excluded from our sample.

The stellar metallicities were taken from Cayrel de Strobel et al. (2001). Additionally, we took $u b v y-\mathrm{H} \beta$ photometry from the catalogue of Hauck \& Mermilliod (1998). We calculated $[\mathrm{Fe} / \mathrm{H}]$ from these data adopting the calibration by Schuster \& Nissen (1989). We define the AMRs using spectroscopic metallicities of Cayrel de Strobel et al. (2001) as 'spectroscopic AMR' and the AMR using photometric metallicity derived from the data of Hauck \& Mermilliod (1998) as 'photometric AMR'. Abundances of $\mathrm{Ca}$ were taken from many other articles. Detailed bibliographies are given in Ibukiyama \& Arimoto (2002, hereafter IA).

We adopted radial velocities from the Hipparcos Input Catalogue, Barbier-Brossat et al. (1994), WEB catalogue, and Malaroda et al. (2001). Proper motions were taken from the Hipparcos Catalogue. The space motions relative to the Sun, $(U, V, W)$, were calculated using Johnson \& Soderblom (1987).

We obtained 4240 sample stars by cross-checking Hipparcos data, the $[\mathrm{Fe} / \mathrm{H}]$ or the $u b v y-\mathrm{H} \beta$ catalogue, and the radial velocity catalogue. We made the sample selection and 1658 stars remained in our sample (see Table 1).

\subsection{Analysis}

We adopted Yonsei-Yale isochrones (Yi et al. 2001) to derive ages by isochrone fitting. We assumed that $[\alpha / \mathrm{Fe}]=0$ and interpolated the isochrones in $\log Z$ with 
the two adjacent metallicities, and derived a set of isochrones with $Z$ and ages from 1.0 to $20.0 \mathrm{Gyr}$. The stellar age was derived by interpolating the grid points of the isochrones on the $M_{\mathrm{V}_{0}}-(B-V)_{0}$ diagram. A schematic view is given in Figure 2 of IA.

We calculated the orbital parameters of sample stars in a similar way to E93 and Nissen \& Schuster (1997). We integrated each star's orbit by assuming constant Galactic potential. We derived the apo-Galactocentric distance projected onto the Galactic plane $R_{\mathrm{a}}$, peri-Galactocentric distance $R_{\mathrm{p}}$, and maximum deviation from the plane $z_{\max }$. We also derived eccentricity $e$, and mean distance $R$. We identified the populations of stars by using their rotational velocities $V$, with respect to the Galaxy centre. The stars

Table 1. The sample stars

\begin{tabular}{lcccr}
\hline & Thin disk & Thick disk & Halo & Total \\
\hline $\begin{array}{l}\text { Photometric }[\mathrm{Fe} / \mathrm{H}] \\
\begin{array}{l}\text { Spectroscopic }[\mathrm{Fe} / \mathrm{H}] \\
\text { (without Ca) }\end{array}\end{array}$ & 1010 & 143 & 16 & 1169 \\
$\begin{array}{l}\text { Spectroscopic }[\mathrm{Fe} / \mathrm{H}] \\
(\text { with Ca) }\end{array}$ & 199 & 58 & 20 & 277 \\
Total & 1382 & 229 & 47 & 1658 \\
\hline
\end{tabular}

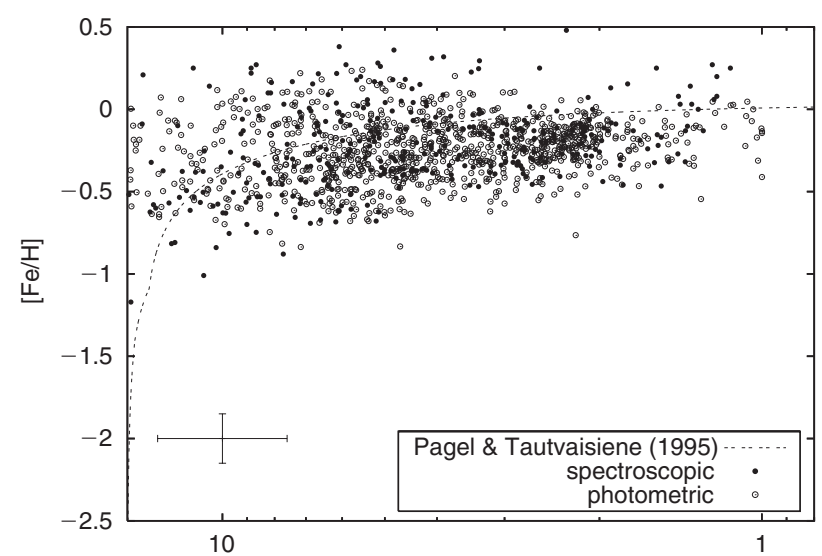

(a)

Age

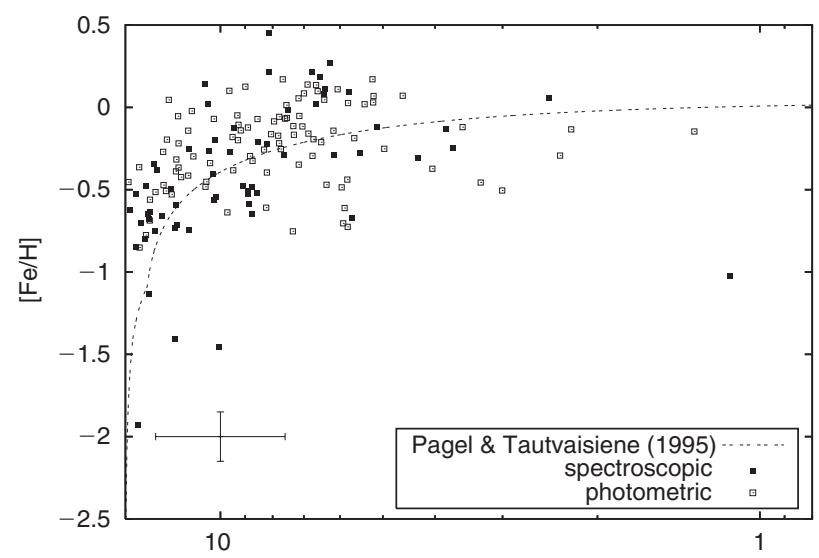

(c)

Age with $V \geq-62 \mathrm{~km} \mathrm{~s}^{-1},-182 \mathrm{~km} \mathrm{~s}^{-1} \leq V<-62 \mathrm{~km} \mathrm{~s}^{-1}$, and $V<-182 \mathrm{~km} \mathrm{~s}^{-1}$ are assigned as thin disk, thick disk, and halo stars, respectively. Following Carney et al. (1989) and Prochaska et al. (2000), stars with $z_{\max }>600 \mathrm{pc}$ are identified with the thick disk component even if their rotational velocities, $V$, are larger than $-62 \mathrm{~km} \mathrm{~s}^{-1}$.

Thin disk stars rotate, on average, around the Galactic centre every 0.2 Gyr. Most of the solar neighbourhood stars are older than $2 \mathrm{Gyr}$ and have rotated more than 10 times since they were formed. When we integrate such stars backward to more than $2 \mathrm{Gyr}$, we observe that they are uniformly distributed in the torus $6-9 \mathrm{kpc}$ from the Galactic centre because of their random motion (see IA, Fig. 4 for details). As a result, the sample stars older than 2 Gyr trace the star formation history in the various regions in the torus, in spite of the fact that they now exist in a region within $100 \mathrm{pc}$ from the Sun. Our sample stars reflect the star formation history not in the narrow sphere with a radius of $100 \mathrm{pc}$ but in the wide torus region whose radius is $6-9 \mathrm{kpc}$ from the Galactic centre.

\section{AMR}

Figure 1(a) shows the AMR of thin disk stars. The line represents the GCE model by PT. The thin disk AMR has
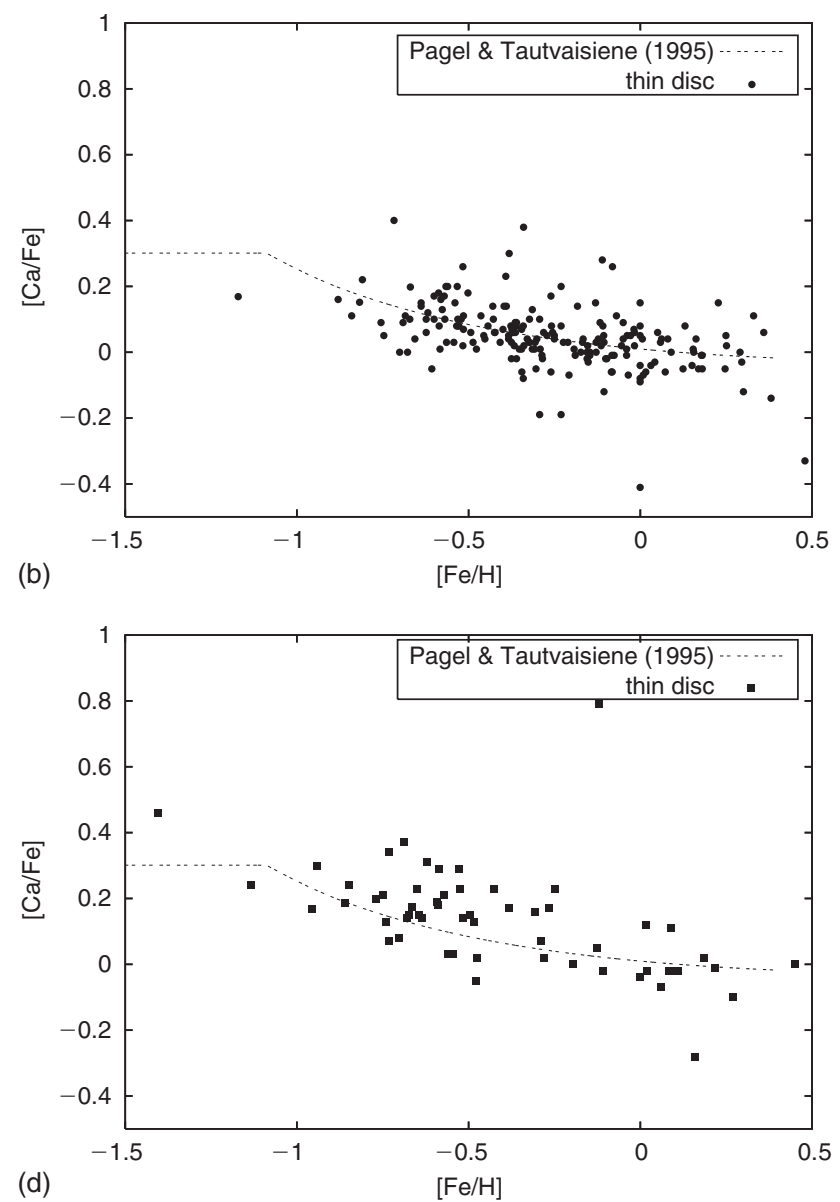

Figure 1 AMRs and abundance patterns: (a) AMR for thin disk; (b) $[\mathrm{Fe} / \mathrm{H}]$ versus $[\mathrm{Ca} / \mathrm{Fe}]$ for thin disk; (c) AMR for thick disk; and (d) $[\mathrm{Fe} / \mathrm{H}]$ versus $[\mathrm{Ca} / \mathrm{Fe}]$ for thin disk. 
a considerable scatter, which is larger than that expected from observational errors. Thin disk stars seem to appear at the beginning of the Galaxy's formation although we are not sure of this feature because such stars have large errors in age determination due to the heavy crowding of isochrones. In our data, the mean metallicity is almost constant and the scatter along the AMR decreases towards younger stars. We see that a similar feature is already apparent in E93 if we consider the stars younger than 10 Gyr. For stars older than $10 \mathrm{Gyr}$, our AMR includes more metal-rich stars than that of E93, and yet it would be difficult to discuss this difference in detail because the age determination of these old stars contains larger errors than that of younger stars and the number of sample stars of E93 is inadequate. There are several features that were not clearly seen in E93's AMR: (1) the upper envelope of the AMR is remarkably flat at a level similar to the solar metallicity for all times from $14 \mathrm{Gyr}$ to $1 \mathrm{Gyr}$; and (2) although the AMR tends to converge on the point $[\mathrm{Fe} / \mathrm{H}] \simeq+0.3$ at 1.6 Gyr in E93's AMR, we found that a fairly large scatter in $[\mathrm{Fe} / \mathrm{H}]$ still exists even at $1 \mathrm{Gyr}$.

Figure 1(b) shows the $[\mathrm{Ca} / \mathrm{Fe}]-[\mathrm{Fe} / \mathrm{H}]$ relation for thin disk stars. The PT model, shown as a solid line, shows good agreement with the average value of our data. The dispersion of $[\mathrm{Ca} / \mathrm{Fe}]$ along the model line is, however, larger than that expected from the uncertainty of observation.

Figure 1(c) presents the thick disk AMR. We identify three features: (1) the bulk of thick disk stars are older than $5 \mathrm{Gyr}$ (the average age of thick disk stars is $8.2 \mathrm{Gyr}$ ); (2) the mean metallicity of thick disk stars is $\langle[\mathrm{Fe} / \mathrm{H}]\rangle \sim-0.5$ and the spread in $[\mathrm{Fe} / \mathrm{H}]$ ranges from $[\mathrm{Fe} / \mathrm{H}]=-1.0$ to solar (this feature is consistent with previous research, see Gilmore \& Wyse 1985; Carney et al. 1989; Layden 1995a, 1995b); (3) the scatter along the AMR is larger than that for a thin disk.

Figure 1(d) shows the $[\mathrm{Ca} / \mathrm{Fe}]-[\mathrm{Fe} / \mathrm{H}]$ diagram for thick disk stars. The dotted line in Figure 1(d) represents the best fit model for the chemical evolution of the outer thin disk by PT. Clearly, Figure 1(d) shows that the thick disk stars are more $\alpha$-enhanced than the thin disk stars at the same $[\mathrm{Fe} / \mathrm{H}]$ value. This trend has already been reported by Gratton et al. (2000) and Prochaska et al. (2000). Interestingly, bulge stars of the Galaxy show a similar trend of $\alpha$-enhancement and are believed to have experienced rapid star formation history (McWilliam \& Rich 1994; Rich \& McWilliam 2000). The $\alpha$-enhancement of thick disk stars, therefore, may also be understood as the result of rapid star formation.

\section{Discussion}

\subsection{Abundance Gradient}

In order to discuss the radial abundance gradient of the Galaxy, it is not appropriate to use the present position of the stars as mean positions of the stellar orbits. We used, instead, $R \equiv\left(R_{\mathrm{p}}+R_{\mathrm{a}}\right) / 2$ for the characteristic distance from the Galactic centre (E93). We also employed $z_{\text {max }}$ for the characteristic distance from the Galactic plane.
Figures 2(a) and 2(b) present the $[\mathrm{Fe} / \mathrm{H}]-R$ and $[\mathrm{Fe} / \mathrm{H}]-$ $z_{\max }$ relation for thin disk stars, respectively. Both sets of data also show no strong correlation between $R / z_{\max }$ and $[\mathrm{Fe} / \mathrm{H}]$. Figures $2(\mathrm{c})$ and $2(\mathrm{~d})$ represent the $[\mathrm{Fe} / \mathrm{H}]-R$ and $[\mathrm{Fe} / \mathrm{H}]-z_{\max }$ relation for thick disk stars, respectively. Correlation between $R$ and $[\mathrm{Fe} / \mathrm{H}]$ is small. As for $[\mathrm{Fe} / \mathrm{H}]$ $z_{\max }$, we see that the thick disk stars more distant from the Galactic plane tend to be more metal poor, namely, a vertical abundance gradient is seen in the thick disk. Comparing the thin disk stars and thick disk stars with $100 \mathrm{pc}<$ $z_{\max }<600 \mathrm{pc}$, the thick disk stars are clearly more metal deficient than the thin disk stars. This phenomenon is naturally understood if we surmise that these two kinds of stellar groups have a different origin: the thick disk stars located at the distance $z_{\max }>100 \mathrm{pc}$ formed intrinsically in the region distant from the Galactic plane while the thin disk stars formed near the Galactic plane and drifted vertically to $100 \mathrm{pc}$ away from their original birthplace.

\subsection{Formation Process for a Thick Disk}

We here discuss how a thick disk formed in the Galaxy. In summary, thick disk stars show three important features: (1) they are older than 5 Gyr; (2) they show vertical abundance gradients; and (3) they have different abundance patterns compared with their thin disk counterparts. To date, thick disk formation scenarios, such as monolithic collapse (Larson 1976; Jones \& Wyse 1983), dynamical heating (Noguchi 1998), major merger and accretion of dwarf galaxies (Quinn \& Goodman 1986) have been proposed. A major merger should invoke a strong peak in age distribution. It is also widely believed that a merger will work in reducing the vertical abundance gradient. Our research does not, therefore, support thick disk formation with a major merger. If a thick disk is formed from heating of the thin disk, the star formation history of a thick disk should be similar to that of the thin disk and the similar star formation history essentially produces the similar $[\mathrm{Ca} / \mathrm{Fe}]-[\mathrm{Fe} / \mathrm{H}]$ relation. A heating mechanism is, therefore, unlikely to explain the thick disk formation. The other two theories both account for our results.

\subsection{Scatter in the AMR for the Thin Disk}

We tried to explain the scatter along the AMR by simple extension of the one-zone model derived by PT. We examined two possibilities.

(1) Metallicity-orbit relation: The solar neighbourhood stars consist of a mixture of stars born at different places with different orbits. This fact may account for the scatter along the thin disk AMR (E93; PT; Prantzos \& Boissier 2000).

(2) Inhomogeneous effective yield and star formation: The effective yield can be different from place to place although the true yield is homogeneous, because the supernova ejecta are inhomogeneous due to axisymmetric explosions of rotating massive stars (Maeda et al. 2002). Even if the explosions are isotropic, the ejecta may stochastically contaminate the surroundings, unless the ISM is distributed uniformly. It is also possible that 

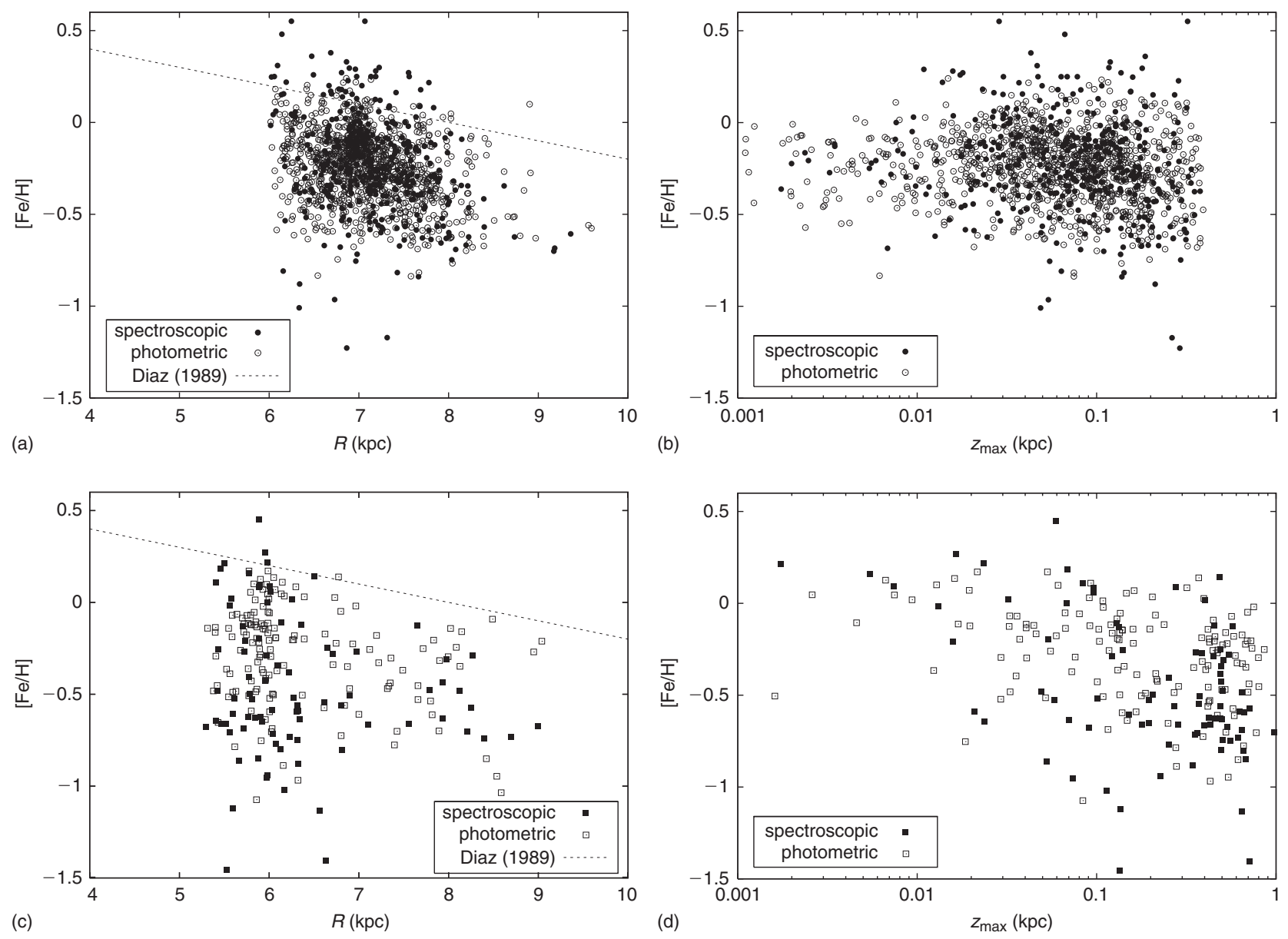

Figure 2 The abundance gradients: (a) $[\mathrm{Fe} / \mathrm{H}]$ versus $R$ for a thin disk; (b) $[\mathrm{Fe} / \mathrm{H}]$ versus $z_{\max }$ for a thin disk; (c) [Fe/H] versus $R$ for a thick disk; and (d) $[\mathrm{Fe} / \mathrm{H}]$ versus $z_{\max }$ for a thick disk. Dashed lines in panels (a) and (c) represent observations of H II regions (Díaz 1989).

local inhomogeneity of the star formation rate produces the scatter.

Detailed analyses are given in IA. Comparing these two hypotheses and our data, we conclude that such a simple modification of the one-zone model cannot explain the scatter along the AMR, age $-[\mathrm{Ca} / \mathrm{Fe}]$ relation, and $[\mathrm{Ca} / \mathrm{Fe}]-[\mathrm{Fe} / \mathrm{H}]$ relations simultaneously.

\section{Summary and Conclusion}

The AMR and orbital parameters are newly derived for 1658 solar neighbourhood stars. The sample stars include 1382 thin disk, 229 thick disk, and 47 halo stars, according to their orbital parameters. Notably, the thin disk AMR shows a considerable scatter along the one-zone GCE model. The scatter along the AMR exists even if stars with the same orbits are selected. We examined a simple extension of the one-zone GCE models and found such extensions of the one-zone GCE model cannot account for the scatter in the age- $[\mathrm{Fe} / \mathrm{H}]-[\mathrm{Ca} / \mathrm{Fe}]$ relation simultaneously. In our work, the scatter along the AMR for thin disk stars has been confirmed for far larger samples (1382 stars), which is more than five times larger than in the previous work of E93. We conclude that this scatter, which should be accounted for by any model of the Galaxy, is one of the most important and essential features of the formation and evolution of the Galaxy. On the other hand, the AMR for thick disk stars shows that star formation terminated $8 \mathrm{Gyr}$ ago in the thick disk. We reconfirmed the trend that the thick disk stars are more Ca-rich than the disk stars with the same $[\mathrm{Fe} / \mathrm{H}]$, which has already been reported by Gratton et al. (2000) and Prochaska et al. (2000). Thick disk stars show a vertical abundance gradient. The thick disk AMR, thick disk vertical gradient, and thick disk $[\mathrm{Ca} / \mathrm{Fe}]-[\mathrm{Fe} / \mathrm{H}]$ relation support monolithic collapse and/or accretion of satellite dwarf galaxies as a thick disk formation scenario.

\section{Acknowledgement}

I am grateful to Brad Gibson for inviting me to such an exciting meeting. I acknowledge Professor Arimoto for many discussions, and thank JSPS for financial support.

\section{References}

Barbier-Brossat, M., Petit, M., \& Figon, P. 1994, A\&AS, 108, 603 Carney, B. W., Latham, D. W., \& Laird, J. B. 1989, AJ, 97, 423

Cayrel de Strobel, G., Soubiran, C., \& Ralite, N. 2001, A\&A, 373,159

Díaz, A. I. 1989, in Evolutionary Phenomena in the Galaxies, eds. J. E. Beckman \& B. E. J. Pagel (Cambridge: Cambridge University Press), 377 
Edvardsson, B., Andersen, J., Gustafsson, B., Lambert, D. L., Nissen, P. E., \& Tomkin, J. 1993, A\&A, 275, 101 (E93)

ESA 1997, The Hipparcos and Tycho Catalogues, SP-1200

Gilmore, G., \& Wyse, R. F. G. 1985, AJ, 90, 2015

Gratton, R. G., Carretta, E., Matteucci, F., \& Sneden, C. 2000, A\&A, 358,671

Hauck, B., \& Mermilliod, M. 1998, A\&AS, 129, 431

Ibukiyama, A., \& Arimoto, N. 2002, A\&A, 394, 927 (IA)

Johnson, D. R. H., \& Soderblom, D. R. 1987, AJ, 93, 864

Jones, B. J. T., \& Wyse, R. F. G. 1983, A\&A, 120, 165

Larson, R. 1976, MNRAS, 176, 31

Layden, A. C. $1995 \mathrm{a}, \mathrm{AJ}, 110,2288$

Layden, A. C. 1995b, AJ, 110, 2312

Maeda, K., Nakamura, T., Nomoto, K., Mazzali, P. A., Patat, F., \& Hachisu, I. 2002, ApJ, 565, 405

Malaroda, S., Levato, H., \& Galliani, S. 2001, Complejo Astronomico El Leoncito
McWilliam, A., \& Rich, R. M. 1994, ApJS, 91, 749

Nissen, P. E., \& Schuster, W. J. 1997, A\&A, 326, 751

Noguchi, M. 1998, Nature, 392, 253

Pagel, B. E. J., \& Tautvaišienè, G. 1995, MNRAS, 276, 505 (PT)

Prantzos, N., \& Boissier, S. 2000, MNRAS, 313, 338

Prochaska, J. X., Naumov, S. O., Carney, B. W., McWilliam, A., \& Wolfe, A. M. 2000, AJ, 120, 2513

Quinn, P., \& Goodman, J. 1986, ApJ, 309, 472

Rich, R. M., \& McWilliam, A. 2000, in SPIE Proc 4005, ed. J. Bergeron (Washington: SPIE), 150

Schuster, W. J., \& Nissen, P. E. 1989, A\&A, 221, 65

Twarog, B. A. 1980, ApJ, 242, 242

Yi, S., Demarque, P., Kim, Y.-C., Lee, Y.-W., Ree, C. H., Lejeune, T., \& Barnes, S. 2001, ApJS, 136, 417 